\title{
MÍNIMO CONSUMO DE CARBONO NA REDUÇÃO EM ALTO-FORNO*
}

Enrique Roy Dionisio Calderon ${ }^{1}$ José Carlos D'Abreu²

\section{Resumo}

O presente trabalho mostra a concepção de um modelo que permite gerar uma superfície de mínimo consumo de redutor (carbono) em Altos-Fornos. No seu desenvolvimento foram consideradas as condições de equilíbrio das reações de redução, os balanços de massa e de energia e parâmetros operacionais. Sua aplicação à redução em Altos Fornos é exemplificada para situações reais, baseada em banco de dados operacionais de uma usina siderúrgica. Foi possível, a partir de planos de corte nestas superfícies, gerar diagramas binários do tipo CR-RD (Consumo de Redutor-Fração de Redução Direta), com possibilidade de serem gerados "on-line" no processo. Dentre os resultados obtidos, destaca-se a identificação de inviabilidades termodinâmicas importantes do forno. Dentre elas o modelo permitiu, através de seus "outputs" gráficos, prever e acompanhar o fenômeno de "gelada de forno", conferindo desta forma maior segurança operacional ao processo.

Palavras-chave: Alto-forno; Coque "rate"; Reduções direta e indireta, Balanços de massa e energia.

\section{MINIMUM CARBON CONSUMPTION IN THE REDUCTION PROCESS IN BLAST FURNACE}

\section{Abstract}

In this paper a model is presented with the objective to generate the surface of minimum consumption of reductant (carbon) for Blast furnaces. In developing the model the equilibrium conditions of the reduction, the mass and energy balances, and the operational parameters, were considered. Its application to the blast furnaces is exemplified for real situations, based on operational database of a steelworks.It was possible, from cutting plans on these surfaces, generate binary diagrams of RCDR type (Reductant Consumption-fraction Direct Reduction), with the possibility of being generated "on-line" in the process. Among the results, there is identification of infeasibility important thermodynamic the furnaces. The model allows through its outputs graphics follow the dynamics of phenomena such as "cold furnaces" to provide greater operational safety to the process.

Keywords: Blast furnace; Coke rate; Direct and indirect reductions; Mass and energy balances.

1 D Sc, Eng. Químico pesquisador do Grupo de Siderurgia do Departamento de Engenharia Química e de Materiais (DEQM) da Pontifícia Universidade Católica do Rio de Janeiro/PUC-Rio, Brasil.

2 D Sc, Professor Emérito do Departamento de Engenharia Química e de Materiais (DEQM) da Pontifícia Universidade Católica do Rio de Janeiro/PUC-Rio, Brasil. 


\section{INTRODUÇÃO}

Em 2015 a produção mundial de aço alcançou mais de 1,6 bilhões de toneladas dos quais $65 \%$ passaram pelo Alto-Forno (AF). No Brasil, a produção foi de 33,2 milhões de ton. de aço, sendo que $85 \%$ foram devido ao Alto-Forno [11,13], o AF é o reator que ainda se constitui na melhor opção para obtenção de ferro gusa ${ }^{[11]}$. As circunstâncias operacionais nos dias de hoje, muito afetadas por custos mais altos, matérias primas com qualidades inferiores, crise financeira internacional persistente e uma capacidade ociosa elevada, tornam cada vez mais importante o desenvolvimento de métodos e procedimentos que ajudem a enfrentar os principais fatores influentes no custo do ferro gusa ${ }^{[1,4,5]}$.

O carbono é o principal insumo usado como redutor dos óxidos de ferro no alto forno. Como seu emprego, via coque ou carvão vegetal, sempre representou significativa percentagem no custo do gusa, manter seu consumo em níveis ótimos sempre constituiu meta importante. Portanto, é fundamental conhecer os limites que satisfaçam as restrições termodinâmicas e assegurem o provimento da quantidade de carbono para a estequiometria e a demanda de energia das reações de redução, nas condições específicas de cada processo ${ }^{[4,7]}$.

Neste contexto, é apresentado o desenvolvimento de um modelo que gera uma superfície de mínimo consumo de carbono-SMCC, com a finalidade de contribuir na tomada de decisão e análise da condução do processo. Esta superfície foi concebida atendendo essencialmente os equilíbrios das reações e os balanços de massa e de energia, de forma a satisfazer a demanda total de carbono para a redução. No presente trabalho é apresentada sua aplicação a casos reais relativos a um alto forno a coque $[2,3,6,8,9,10]$.

\section{DESENVOLVIMENTO}

Os equacionamentos dos balanços estagiados de massa, energia, e global do AltoForno foram feitos com base nos algoritmos e diagramas clássicos de Michard e Rist, para as regiões de preparação e elaboração, referentes as trocas térmicas e de oxigênio ${ }^{[13]}$.

A redução indireta, $\mathrm{RI}$, dos óxidos ferrosos com o gás redutor $\mathrm{CO}$, gerando $\mathrm{CO}_{2}$, e redução direta, $\mathrm{RD}$, resultante da sequencia redução indireta- reação de Boudouard conduziu ao balanço global de massa, conforme a equação 1:

$$
X+Y+V=(1-R D)(n+G)\left(\frac{a+b}{b+d}\right)
$$

Onde X: Átomos de carbono para Redução Direta;

Y: Átomos de carbono existentes no gás $\mathrm{CO}$ produto da reação de Boudouard;

V: Átomos de carbono existentes no gás CO produto da redução da água;

RD: Fração de Redução Direta;

n: Átomos de oxigênio presentes nos óxidos de ferro, a serem reduzidos;

G: Átomos de oxigênio presentes nos óxidos não ferrosos, a serem reduzidos;

a: Átomos de oxigênio presentes no CO do gás de topo;

b: Átomos de oxigênio presentes no gás $\mathrm{CO}_{2}$ do gás de topo;

d: Átomos de oxigênio presentes no $\mathrm{H}_{2} \mathrm{O}$ do gás de topo. 
$\mathrm{Na}$ zona de elaboração, o carbono total necessário para satisfazer o equilíbrio, é dado por:

$$
C E Q=\frac{1}{K_{C Q}}\left[1.05+\omega+G-\left(X+\frac{K_{H} \times Z}{K_{H}+1}\right)\right]
$$

Onde CEQ: Carbono necessário para satisfazer o equilíbrio;

$\mathrm{Kco}$ : Constante de equilíbrio da redução de $\mathrm{FeO}$ pelo $\mathrm{CO}$;

$\omega$ : $\quad$ Fator ômega, ou desvio de Rist;

$\mathrm{K}_{\mathrm{H}}$ : Constante de equilíbrio da redução do $\mathrm{FeO}$ pelo $\mathrm{H}_{2}$;

Z: $\quad$ Átomos de hidrogênio produzidos pela redução da água.

O carbono necessário para satisfazer as demandas térmicas do balanço de energia das regiões de elaboração e de combustão é dado por:

$$
G E T=\frac{C G E}{C G S}
$$

Onde:

CBT: Quantidade de carbono necessário para satisfazer a demanda térmica, por átomo de ferro;

CGE: Calor demandado pela zona de elaboração;

CGS: Calor gerado na zona de combustão.

O equacionamento da superfície de mínimo consumo de carbono foi realizado levando-se em conta que, em altas taxas de Redução Direta, o equilíbrio da redução da wüstita via intermediário gasoso define o consumo de carbono. Entretanto, sendo a Redução Direta endotérmica, ela também demandará carbono para satisfazer esses requisitos térmicos. Por outro lado, quando as taxas de Redução Indireta forem altas, existirá diminuição daquela demanda térmica e, portanto, menos carbono para aquele fim. Porém, devido ao equilíbrio estequiométrico da redução indireta, haverá aumento na demanda de CO.

Finalmente, o equacionamento da superfície de mínimo consumo de carbono, como função das variáveis $\mathrm{R}$ e RD, foi possível, conforme representado pelas equações (4) e (5):

\section{Quando: CEQ>CBT}

$$
C C_{\Theta Q}=C E Q \times K_{C Q} \times\left[\frac{\pi}{K_{H}}+1\right]+C_{G V S A}
$$

Ou, quando: $\mathrm{CBT}>\mathrm{CEQ}$

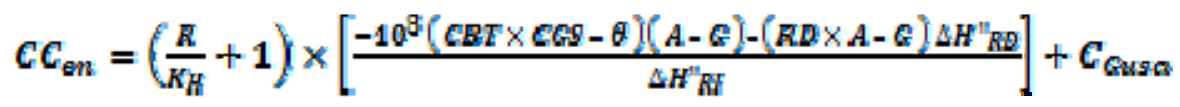

Onde:

CCeq: Carbono mínimo consumido no processo, considerando o equilíbrio;

CCen: Carbono mínimo consumido, considerando o balanço térmico;

Cgusa: Carbono contido no gusa;

R: $\quad$ Relação entre $\mathrm{CO}, \mathrm{CO}_{2}$ e $\mathrm{H}_{2}, \mathrm{H}_{2} \mathrm{O}$, no gás de topo; 
A: $\quad$ Fator igual a $1,05+\omega+G$;

$\theta$ : $\quad$ Função contendo perdas térmicas, calor contido no gusa e na escória, e as entalpias das reduções dos óxidos não ferrosos e reduções pelo $\mathrm{H}_{2}$;

$\Delta \mathrm{H}_{\mathrm{RD}}$ : Entalpia para a Redução Direta;

$\Delta \mathrm{H}_{\mathrm{RI}}$ : Entalpia para a Redução Indireta.

Constituída na realidade por duas superfícies de consumo de carbono, ou seja, uma gerada pelo equilíbrio, expressa pela eq. (4), e a outra pelo balanço térmico, representada pela eq. (5), obteve-se a superfície resultante, a qual deverá sempre satisfazer o maior valor entre $\mathrm{CC}_{e q}$ e $\mathrm{CC}_{e n}$, ou seja, atender a lógica da eq. (6):

$$
\mathrm{CC}_{\text {eff }}=\left\{\mathrm{CC}_{\mathrm{eq} y}, \mathrm{CC}_{\mathrm{en}}\right\}_{\text {supremg } \forall \mathrm{K}, \mathrm{RD}}
$$

Onde $\mathrm{CC}_{\text {eff }}$ : É o Carbono mínimo consumido resultante, expresso como Consumo de Carbono efetivo (at-C/at-Fe).

A figura 1 ilustra a superfície de mínimo consumo de carbono, onde os pontos acima da mesma representam as condições termodinamicamente viáveis do processo, e os pontos abaixo as condições de inviabilidade da redução.

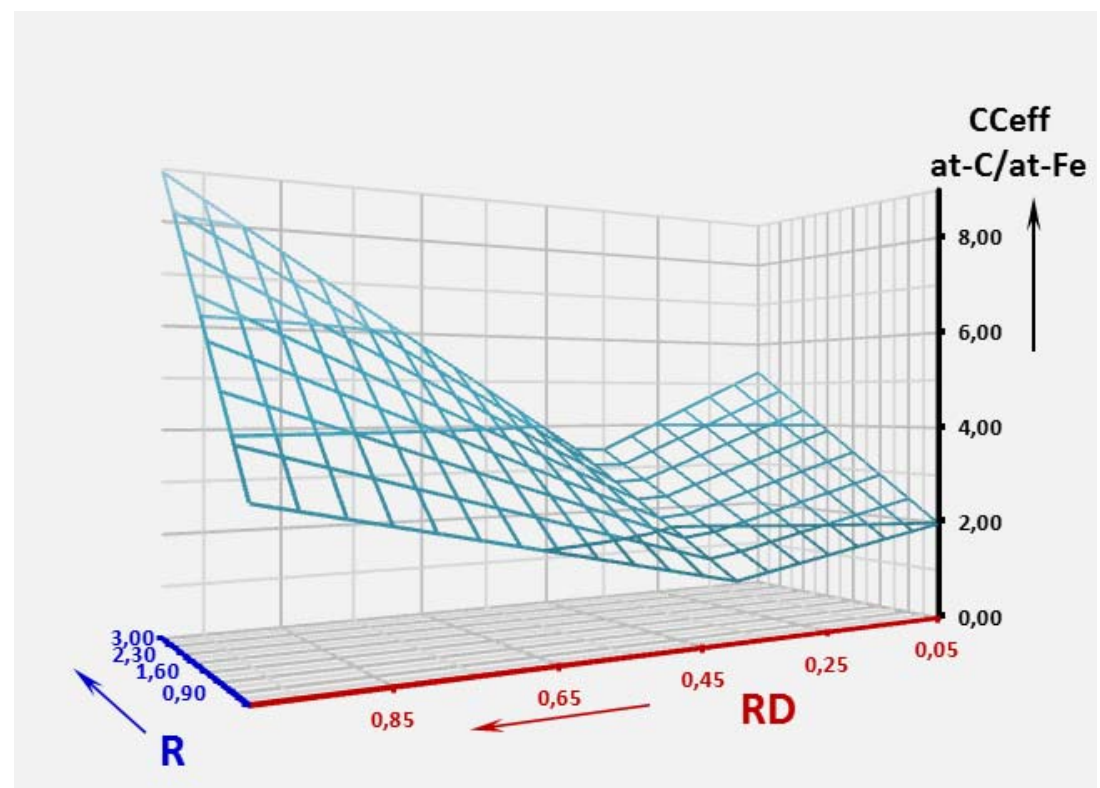

Figura 1. Diagrama da superfície de mínimo consumo de carbono nos eixos: CCeff $x$ R x RD,

\section{RESULTADOS E DISCUSSÕES}

Com a finalidade de exemplificar a aplicação do diagrama em condições reais, foram feitos cortes na SMCC por um plano, este definido para um certo valor da variável $\mathrm{R}$ conhecido da operação, como mostrado na figura 2a. Estes cortes definiram, em consequência, curvas bidimensionais do tipo CR-RD (Consumo de RedutorRedução Direta), como representado na figura $2 \mathrm{~b}$, na qual o consumo efetivo de carbono ( $\left.\mathrm{CC}_{\text {eff }}\right)$ esta apresentado em termos de Coque, atendida pela eq. (7) abaixo (9). 


$$
C R=214,869 \times C C_{\text {eff }} \times\left(\frac{q_{C F e}}{C_{\text {fixe }}}\right)+L A P O
$$

Onde LAPO: representa as quantidades de pó de coletor e lama, em kg de Coque/tonelada de gusa.
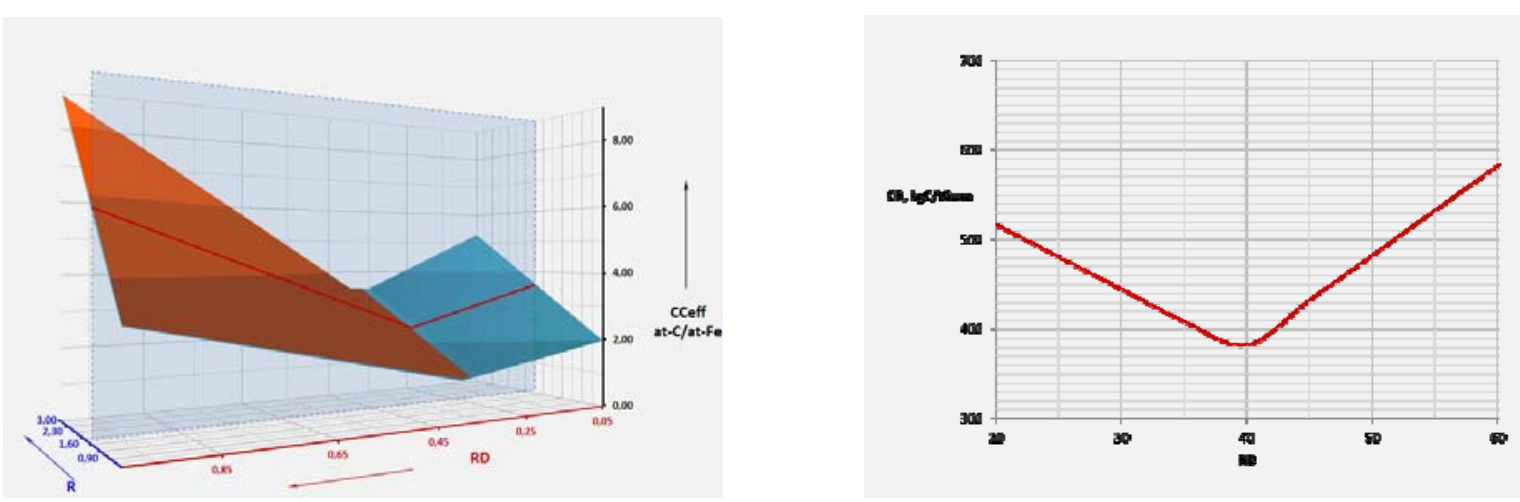

Figura 2a. Plano de corte no diagrama da SMCC (esquerda). Figura 2b, (direita). Curva de Demanda de Carbono Cumprindo as Restrições do Equilíbrio e Balanço Térmico (para um valor de $R=1,012$ ).

\subsection{Estudo de Casos}

Para fins de validação do modelo, foram selecionadas operações reais de um AltoForno a coque, conforme mostrado nas tabelas 1 e 2 . Duas representando operações diárias normais (operações 1 e 2), na tabela 1, e duas outras, a 3 e 4 , relativas a registros de operações reconhecidamente anormais, devido a problemas de esfriamento do forno, ("geladas"), ocorridas num intervalo de cerca de 4 horas.

Tabela 1, dados das operações normais

\begin{tabular}{|l|l|l|l|l|l|l|l|l|l|}
\hline \multicolumn{3}{|c|}{ OPERAÇÃO } & \multicolumn{9}{c|}{ MODELO } & \multicolumn{2}{|l|}{$\begin{array}{l}\text { Desvio da SMCC: } \\
\text { Excesso (+) ou } \\
\text { deficiência (-) de }\end{array}$} \\
\hline$N^{\circ}$ & $R$ & $\omega$ & CRop & RDop & CRRDop & CRótimo & RDótimo & $\begin{array}{l}\text { Do } \\
\text { CR Rop }\end{array}$ & $\begin{array}{l}\text { do } \\
\text { CRótimo }\end{array}$ \\
\hline 1 & 1,0801 & 0,0132 & 464,5 & 44,30 & 442,920 & 397,96 & 40,0 & $+21,580$ & $+66,54$ \\
\hline 2 & 1,0122 & 0,0094 & 464,5 & 41,78 & 400,178 & 382,36 & 39,8 & $+64,322$ & $+82,14$ \\
\hline
\end{tabular}

Tabela 2, dados das operações com resfriamento do forno

\begin{tabular}{|l|l|l|l|l|l|l|l|l|l|}
\hline \multicolumn{3}{|c|}{ OPERAÇÃO } & \multicolumn{9}{c|}{ MODELO } & \multicolumn{2}{|l|}{$\begin{array}{l}\text { Desvio da SMCC: } \\
\text { Excesso (+) ou } \\
\text { deficiência (-) de }\end{array}$} \\
\hline$N^{\circ}$ & $R$ & $\omega$ & CRop & RDop & CRRDop & CRótimo & RDótimo & $\begin{array}{l}\text { do } \\
\text { CRRDop }\end{array}$ & $\begin{array}{l}\text { do } \\
\text { CRótimo }\end{array}$ \\
\hline 3 & 1,1628 & 0,0122 & 473,0 & 50,34 & 573,232 & 444,63 & 39,0 & $-100,23$ & $+28,37$ \\
\hline 4 & 0,7557 & 0,0272 & 472,3 & 54,43 & 485,518 & 358,75 & 39,8 & $-13,22$ & $+113,6$ \\
\hline
\end{tabular}

Nas figuras 3 e 4 são apresentados os cortes CR-RD da superfície de mínimo consumo de carbono: 
A figura 3 ilustra o diagrama CR-RD das operações 1 e 2, com as indicações dos pontos operacionais reais, calculados via a metodologia de Rist (em azul) e seus afastamentos da fronteira de viabilidade termodinâmica (curva vermelha).
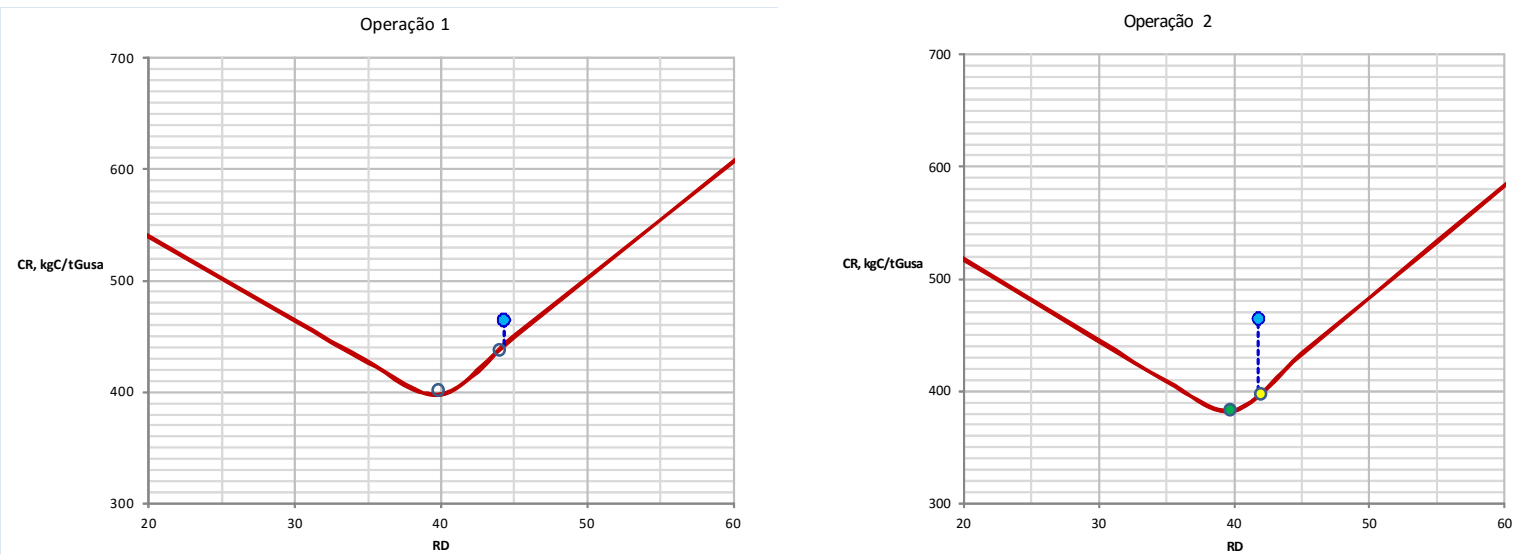

Figura 3. Diagramas CR-RD para operações normais do Alto-Forno (operações 1 e 2).

A figura 4, por sua vez, apresenta os diagramas CR-RD para as condições anormais 3 e 4, com as indicações dos pontos operacionais reais (em azul) e seus afastamentos da fronteira de viabilidade, os quais se colocaram nitidamente abaixo da linha de consumo mínimo de coque.
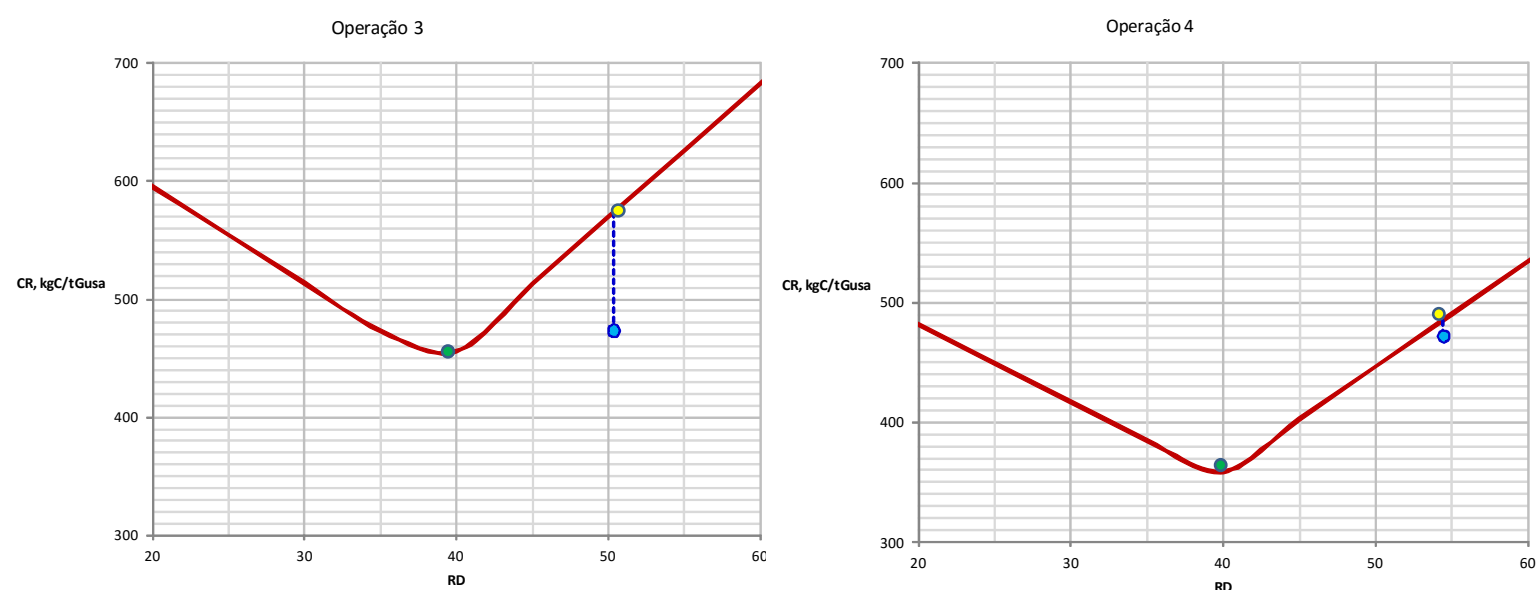

Figura 4. Diagrama CR-RD para operações anormais do Alto-Forno, operação $3(01: 00 \mathrm{~h})$ e operação 4(05:00 h).

\section{CONCLUSÕES}

1) A SMCC, como concebida e gerada no presente trabalho, permitiu a interpretação geométrica tridimensional da fronteira de viabilidade termodinâmica do processo de redução, ou seja, a definição de operações normais e anormais, dependendo da localização dos pontos operacionais se situarem acima ou abaixo da SMCC, respectivamente;

2) Para maior praticidade da função resposta, foram considerados no sistema tridimensional, cortes planares correspondentes a valores constantes da variável R, característica do gás de topo, definindo os diagramas CR-RD;

3) Nos diagramas bidimensionais, CR-RD, as análises operacionais de controle e manutenção da viabilidade do processo de redução passaram a depender 
essencialmente da determinação do ponto operacional efetivo do processo e de sua representação gráfica nos diagramas;

4) Evidenciou-se que a otimização termodinâmica ficou representada pelo ponto de interseção das duas curvas do diagrama bidimensional, indicando que a região objetivada pela operação deverá se localizar acima e o mais possível no seu entorno;

5) A validação do modelo desenvolvido utilizou dados de relatórios diários de produção de uma usina siderúrgica integrada nacional, considerando períodos de marchas regulares e anormais;

Para o caso A, relativo a operações normais, confirmou-se suas viabilidades termodinâmicas, ficando seus pontos operacionais acima das curvas fronteiras;

Para o caso B, relativo a operações anormais, confirmou-se suas inviabilidades termodinâmicas, tendo em vista seus pontos operacionais abaixo das curvas fronteiras;

6) No momento, o modelo esta sendo testado como uma ferramenta de controle efetivamente operacional, implementada "on-line" aos bancos de dados formados pelas aquisições automáticas e contínuas da operação de um Alto-Forno. Esta implementação, permitirá prever, com uma antecedência segura de horas, a tendência da operação estar-se encaminhando para inviabilidades termodinâmicas, viabilizando, assim, a tomada antecipada das medidas corretivas necessárias.

\section{Agradecimentos}

Ao Grupo de Siderurgia da PUC-Rio/DEQM pelo apoio na pesquisa.

\section{REFERÊNCIAS}

1 ABM. Introdução a Siderurgia-coleção de livros metalurgia, Materiais e Mineração. $1^{a}$ Edição. São Paulo 2011.

2 APARECIDO C. WELLINGTON, Aplicação de um método de Simulação-otimizacão na cadeia produtiva de minérios de ferro, Dissertação de Mestrado. EPUSP, Curso de Pós-Graduação em Engenharia de Sistemas Logísticos, agosto, Setembro de 2007.

3 BILIK J.; KRET J.; BEER H.: Application of The Simulating Mathematical Models for Decreasing of The Blast Furnace Fuel Rate; disponivel em: http://www.ams.tuke.sk/data/ams online/1998/number1/mag07.pdf acessado em Nov 2015.

4 DA SILVEIRA RIZZO, ERNANDES MARCOS. Introdução aos processos Siderúrgicos. São Paulo 2005.

5 DA SILVEIRA RIZZO, ERNANDES MARCOS. Processo de Fabricação de ferro-gusa em Alto-Forno. ABM. São Paulo 2009.

6 DE AGUIAR MELGAÇO JR., HÉLIO. Aplicação dos métodos gráficos de Rist e Reichardt no controle operacional de Altos Fornos a coque. Dissertação de Mestrado. PUC-RIO, Curso de Pós-Graduação em Engenharia Metalúrgica (orientador: José Carlos D'ABREU). Setembro de 1985.

7 DE ARAÚJO, L.A. Manual de siderurgia. $1^{\circ}$ volume. São Paulo: Editora Arte \& Ciência, 1997.

8 DE CASTRO, L.A., Desenvolvimento De Um Modelo de Controle de Processo Para Altos-Fornos A Carvão Vegetal, Tese de Doutorado. UFMG, Curso de Pós-Graduação em Engenharia Metalúrgica e de Minas, agosto, 2002.

9 DE MELO WILSON ROBERTO, Controle do processo de Redução no Alto-Forno II da Cosipa aplicação do diagrama CRRD. Dissertação de Mestrado. PUC-Rio, Curso de 
Pós-Graduação em Engenharia Metalúrgica (orientador José Carlos D’ABREU). Fevereiro de 1987.

10 FUMITADA NAKATANI, T. MUKAI, F. NAKAMURA, Theoretical Consideration on Coke Ratio of the Blast Furnace Operation, Steel Science Portal. Novembro de 1965

11 Instituto Aço Brasil, Relatório de sustentabilidade 2013, disponível em: http://www.acobrasil.org.br/site/portugues/sustentabilidade/relatorio.asp acessado em : 10 Maio 2014.

12 CHICO SANTOS, Cojuntura Econômica, Conjuntura Siderurgia, FGVIBRE, volume 69, $N^{\circ}$ 09, Setembro 2015.

13 DIONISIO CALDERON, ENRIQUE R., Superfície de Mínimo Consumo de Carbono do Processo de Redução em Altos-Fornos. Tese de Doutorado, PUC-Rio, Curso de PósGraduação em Engenharia de Materiais e de Processos Químicos e Metalúrgicos (orientador José Carlos D'ABREU). Fevereiro de 2016. 